\title{
CHARACTERIZATIONS OF THE COMPACTNESS OF RIEMANNIAN MANIFOLDS BY EIGENFUNCTIONS, AND A PARTIAL PROOF OF A CONJECTURE BY HAMILTON
}

\author{
XIANG GAO
}

\begin{abstract}
In this paper, we deal with comparison theorems for the first eigenvalue of the Schrödinger operator, and we present some criteria for the compactness of a Riemannian manifold in terms of the eigenfunctions of its Laplacian. Firstly, we establish a comparison theorem for the first Dirichlet eigenvalue $\mu_{1}^{D}(B(p, r))$ of a given Schrödinger operator. We then prove that, for the space form $M_{K}^{n}$ with constant sectional curvature $K$, the first eigenvalue of the Laplacian operator $\lambda_{1}\left(M_{K}^{n}\right)$ is greater than the limit of the corresponding first Dirichlet eigenvalue $\lambda_{1}^{D}\left(B_{K}(p, r)\right)$. Based on these, we present a characterization of a compact gradient shrinking Ricci soliton locally being an $n$-dim space form by the first eigenfunctions of the Laplacian operator, which gives a generalization of an interesting result by Cheng [4] from 2-dim to $n$-dim. This result also gives a partial proof of a conjecture by Hamilton [7] that a compact gradient shrinking Ricci soliton with positive curvature operator must be Einstein. Finally, we derive a criterion of the compactness of manifolds, which gives a partial proof of another conjecture by Hamilton [6] that, if a complete Riemannian 3-manifold $\left(M^{3}, g\right)$ satisfies the Ricci pinching condition $R c \geq \varepsilon R g$, where $R>0$ and $\varepsilon$ is a positive constant, then it is compact. In fact, our result is also true for general $n$-dim manifolds.
\end{abstract}

1. Introduction and main results. Suppose that $\left(M^{n}, g\right)$ is an $n$ $\operatorname{dim} C^{\infty}$ complete Riemannian manifold, and $\Delta$ denotes the Laplacian operator. If the manifold $M^{n}$ is compact, it is well known that the eigenvalue problem $-\Delta \varphi=\lambda \varphi$ has discrete eigenvalues, which are not

2010 AMS Mathematics subject classification. Primary 35P05, 58G25.

Keywords and phrases. Laplacian operator, Schrödinger operator, eigenvalue, Dirichlet eigenvalue, eigenfunction.

This work was supported by the National Natural Science Foundation of China (NSFC) 11101267, 11301493 and Fundamental Research Funds for the Central Universities.

Received by the editors on May 20, 2014. 
counted according to their multiplicity. We list them as

$$
0=\lambda_{0}\left(M^{n}\right)<\lambda_{1}\left(M^{n}\right) \leq \lambda_{2}\left(M^{n}\right) \leq \cdots,
$$

and call $\lambda_{i}\left(M^{n}\right)$ the $i$ th eigenvalue and a function satisfying $\Delta \varphi_{i}=$ $-\lambda_{i} \varphi_{i}$ the $i$ th eigenfunction.

In this paper, we always assume that $V(x)$ is a non-negative function defined on $M^{n}$, which is assumed to be smooth or at least continuous, and deal with the eigenvalue problem $-\Delta \varphi+V \varphi=\mu \varphi$ for the famous Schrödinger operator $-\Delta+V$. We call $\mu_{i}\left(M^{n}\right)$ the $i$ th eigenvalue and a function satisfying $-\Delta \varphi+V \varphi=-\mu_{i} \varphi$ the $i$ th eigenfunction.

Recall that the first eigenvalue of the Laplacian operator $\lambda_{1}\left(M^{n}\right)$ and the one of the Schrödinger operator $\mu_{1}\left(M^{n}\right)$ for a closed Riemannian manifold $M^{n}$ are respectively defined as follows:

$$
\lambda_{1}\left(M^{n}\right)=\inf _{\phi \in \Omega} \frac{\int_{M^{n}}|\nabla \phi|^{2} d \mu}{\int_{M^{n}} \phi^{2} d \mu} \neq 0,
$$

and

$$
\mu_{1}\left(M^{n}\right)=\inf _{\phi \in H_{1}^{2}\left(M^{n}\right) \phi \not \equiv 0} \frac{\int_{M^{n}}\left(|\nabla \phi|^{2}+V \phi^{2}\right) d \mu}{\int_{M^{n}} \phi^{2} d \mu} \neq 0
$$

where

$$
\Omega=\left\{\phi \in H_{1}^{2}\left(M^{n}\right) \mid \int_{M^{n}} \phi d \mu=0\right\},
$$

and $H_{1}^{2}\left(M^{n}\right)$ is the completed Hilbert space of $C^{\infty}\left(M^{n}\right)$ under the norm

$$
\|\phi\|_{H_{1}^{2}}^{2}=\int_{M^{n}} \phi^{2} d \mu+\int_{M^{n}}|\nabla \phi|^{2} d \mu
$$

It is well known that, for a closed Riemannian manifold with positive Ricci curvature, we have the following famous Lichnerowicz-Obata theorem [9]:

Theorem 1.1 (Lichnerowicz-Obata). Let $\left(M^{n}, g\right)$ be a closed Riemannian manifold satisfying $R c \geq(n-1) K>0$, for some constant $K>0$. Then

$$
\lambda_{1}\left(M^{n}\right) \geq n K,
$$

and the equality holds if and only if $\left(M^{n}, g\right)$ is isometric to the space form $\left(M_{K}^{n}, g_{K}\right)$ with constant sectional curvature $K$, and the corre- 
sponding eigenfunction is

$$
\varphi(x)=A \cos (\sqrt{K} r)+B \sin (\sqrt{K} r)
$$

where $A$ and $B$ are two constants and $r=d(p, x)$.

Then we deal with the first Dirichlet eigenvalue of the Laplacian operator and the Schrödinger operator. Let $B(p, r)$ denote the geodesic ball with center $p$ and radius $r$, and let $B_{K}\left(p^{\prime}, r\right)$ denote the geodesic ball with center $p^{\prime}$ and radius $r$ in the $n$-dim simply connected space form $M_{K}^{n}$ with constant sectional curvature $K$. The first Dirichlet eigenvalue $\lambda_{1}^{D}(B(p, r))$ of the Laplacian operator and the one $\mu_{1}^{D}(B(p, r))$ of the Schrödinger operator for the geodesic ball $B(p, r)$ can be respectively defined as follows:

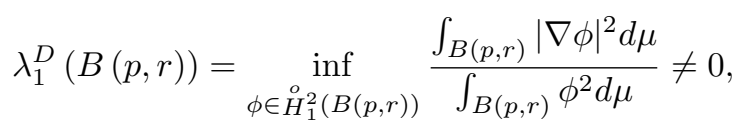

and

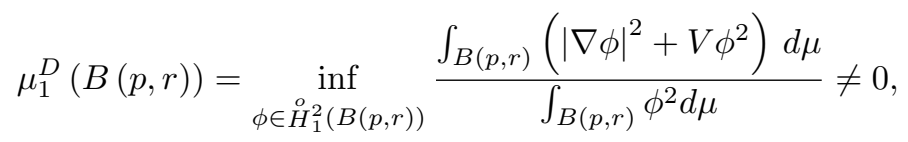

where $\stackrel{o}{H}_{1}^{2}(B(p, r))$ is the completed Hilbert space of $C_{0}^{\infty}(B(p, r))$ under the norm

$$
\|\phi\|_{H_{1}^{2}}^{2}=\int_{B(p, r)} \phi^{2} d \mu+\int_{B(p, r)}|\nabla \phi|^{2} d \mu .
$$

Moreover, using the first Dirichlet eigenvalue $\lambda_{1}^{D}(B(p, r))$ of the Laplacian operator for $B(p, r)$, we can define the first eigenvalue of the Laplacian operator for the complete Riemannian manifold $M^{n}$ as follows:

$$
\lambda_{1}\left(M^{n}\right)=\lim _{r \rightarrow \infty} \lambda_{1}^{D}(B(p, r))
$$

where $B(p, r)$ is the geodesic ball in $M^{n}$.

Recall that, for the $\lambda_{1}^{D}(B(p, r))$, we have the following famous comparison theorem by Cheng $[\mathbf{3}]$ : 
Theorem 1.2 (Cheng). Let $\left(M^{n}, g\right)$ be a complete Riemannian manifold satisfying $R c \geq(n-1) K$, where $K \in \mathbb{R}$. Then

$$
\lambda_{1}^{D}(B(p, r)) \leq \lambda_{1}^{D}\left(B_{K}\left(p^{\prime}, r\right)\right)
$$

and equality holds if and only if $B(p, r)$ is isometric to $B_{K}\left(p^{\prime}, r\right)$.

Corollary 1.3 (Cheng). Let $\left(M^{n}, g\right)$ be a compact Riemannian manifold satisfying $R c \geq 0$. Then

$$
\lambda_{1}\left(M^{n}\right) \leq \lambda_{1}^{D}\left(B\left(p, \frac{d_{M^{n}}}{2}\right)\right) \leq \frac{C_{n}}{d_{M^{n}}^{2}},
$$

where $C_{n}=2 n(n+4)$ and $d_{M^{n}}$ is the diameter of $M^{n}$.

Moreover, for the Schrödinger operator $L=-\Delta+V$ for the manifold $M^{n}$ and $L_{K}=-\Delta+V_{K}$ for the space form $M_{K}^{n}$, where $V_{K}$ is assumed to be smooth and nonnegative. By using Theorem 1.2, we can also derive a similar comparison theorem as follows:

Theorem 1.4 (Comparison theorem for the Schrödinger operator). Let $\left(M^{n}, g\right)$ be a complete Riemannian manifold satisfying $R c \geq(n-1) K$, where $K \in \mathbb{R}$. Then

$$
\mu_{1}^{D}(B(p, r)) \leq \mu_{1}^{D}\left(B_{K}\left(p^{\prime}, r\right)\right)+\max _{x \in B(p, r)} V(x)-\min _{x \in B_{K}\left(p^{\prime}, r\right)} V_{K}(x),
$$

and equality holds if and only if the following conditions are satisfied:

(i) $B(p, r)$ is isometric to $B_{K}\left(p^{\prime}, r\right)$;

(ii) the eigenfunctions corresponding to the first eigenvalues $\lambda_{1}^{D}(B(p, r))$ and $\lambda_{1}^{D}\left(B_{K}\left(p^{\prime}, r\right)\right)$ of the Laplacian operator are, under the isometry from (i), precisely the ones corresponding to the first eigenvalues $\mu_{1}^{D}(B(p, r))$ and $\mu_{1}^{D}\left(B_{K}\left(p^{\prime}, r\right)\right)$ of the Schrödinger operator;

(iii) the function $V(x)$ is a constant on $B(p, r)$ and $V_{K}(x)$ is a constant on $B_{K}\left(p^{\prime}, r\right)$. 
In this paper, based on Theorem 1.2 and the definition of the first Dirichlet eigenvalue of the Laplacian operator, we prove the useful result below, which is also one of our main results:

Theorem 1.5 (Main result I). Let $\left(M_{K}^{n}, g_{K}\right)$ be a space form with constant positive sectional curvature $K$, and let $\lambda_{1}^{D}\left(M_{K}^{n}\right)$ be the first Dirichlet eigenvalue of the Laplacian operator for $M_{K}^{n}$, which is defined as follows:

$$
\lambda_{1}^{D}\left(M_{K}^{n}\right)=\lim _{r \rightarrow \pi / \sqrt{K}} \lambda_{1}^{D}\left(B_{K}\left(p^{\prime}, r\right)\right)
$$

where $\lambda_{1}^{D}\left(B_{K}\left(p^{\prime}, r\right)\right)$ is the first Dirichlet eigenvalue of the Laplacian operator for $B_{K}\left(p^{\prime}, r\right)$. Then we have

$\lambda_{1}^{D}\left(M_{K}^{n}\right)=\lim _{r \rightarrow \pi / \sqrt{K}} \lambda_{1}^{D}\left(B_{K}\left(p^{\prime}, r\right)\right) \leq \lambda_{1}\left(M_{K}^{n}\right) \leq \lambda_{1}^{D}\left(B_{K}\left(p^{\prime}, \frac{\pi}{2 \sqrt{K}}\right)\right)$.

Now we recall the definition of the gradient Ricci soliton as follows $[6]$ :

Definition 1.6 (Gradient Ricci soliton). A complete Riemannian manifold $\left(M^{n}, g\right)$ is called a gradient Ricci soliton if there exists a smooth function $f: M^{n} \rightarrow \mathbb{R}$, such that

$$
R c(g)+\nabla \nabla f+\frac{\varepsilon}{2} g=0,
$$

where $R c$ is the Ricci curvature tensor and $\varepsilon$ is a real constant. Furthermore,

(i) If $\varepsilon<0$, then it is called a shrinking gradient Ricci soliton;

(ii) If $\varepsilon=0$, then it is called a steady soliton;

(iii) If $\varepsilon>0$, then it is called an expanding one.

It is well known that one of the basic problems in Riemannian geometry is to relate curvature and topology. In [1], Böhm and Wilking proved that $n$-dim closed Riemannian manifolds with 2-positive curvature operators are diffeomorphic to spherical space forms, i.e., they admit metrics with constant positive sectional curvature. Moreover, it is a well-known theorem of Tachibana [11] that any compact Einstein 
manifold with positive sectional curvature must be of constant curvature. Note that Einstein manifolds are special Ricci solitons with the constant Ricci potential function; hence, inspired by his own work in $[7,8]$, Hamilton made the following conjecture [7]:

Conjecture 1.7 (Hamilton). A compact gradient shrinking Ricci soliton with positive curvature operator must be Einstein.

Hence, if Conjecture 1.7 is true, then together with Tachibana's theorem, we have:

Conjecture 1.8. A compact gradient shrinking Ricci soliton with positive curvature operator must be a space form with constant positive sectional curvature.

Moreover, for the compact gradient steady or expanding case, Hamilton actually proved a similar result [7]:

Theorem 1.9 (Hamilton). A compact gradient steady or expanding Ricci soliton must be Einstein.

Although the general proof of Conjecture 1.8 is hard, for the special case $S^{2}$, Cheng [4] proved the following interesting result by using tensor analysis and the famous Gauss-Bonnet theorem.

Theorem 1.10 (Cheng). Suppose that $M^{2}$ is homeomorphic to $S^{2}$ and that $\varphi_{1}, \varphi_{2}, \varphi_{3}$ are all first eigenfunctions on $\left(M^{2}, g\right)$ (that is, eigenfunctions corresponding to $\lambda_{1}\left(M^{2}\right)$ ), such that the sum of their squares is equal to a constant. Then $\left(M^{2}, g\right)$ is actually isometric to a sphere with constant sectional curvature.

Remark 1.11. In fact, as mentioned in [4], in Theorem $1.10, m=3$ is the only number such that there are $m$ first eigenfunctions with square sum equal to a constant. Since every nonconstant eigenfunction must vanish somewhere, this rules out that the case $m=1, m=2$ is not possible because, by the results of [5] we have $\varphi_{1}^{-1}(0) \cap \varphi_{2}^{-1}(0) \neq \phi$ whenever $-\Delta \varphi_{i}=\lambda \varphi_{i}, i=1,2, \lambda>0$. Also the results of [5] show 
that if $M$ is homeomorphic to $S^{2}$, then the multiplicity of the first eigenfunction is at most 3 . This shows that $m>3$ is not possible.

Since each manifold with $\operatorname{dim}=2$ is actually an Einstein manifold, which is a special Ricci soliton with a constant Ricci potential function. Then, for the special case of Ricci solitons, we may prove a similar result to Theorem 1.10. In fact, we present a characterization of a Ricci soliton being an $n$-dim space form by the first eigenfunctions of the Laplacian operator, which gives a partial proof of Conjecture 1.8 as follows:

Theorem 1.12 (Main result II). Let $\left(M^{n}, g\right)$ be a compact gradient Ricci soliton with positive Ricci curvature. Suppose that there exists a geodesic ball $B(p, r)$ with center $p$ and radius $r$ such that the eigenfunctions $\left\{\varphi_{i}\right\}_{i=1}^{N}$ corresponding to the first Dirichlet eigenvalue $\lambda_{1}^{D}(B(p, r))$ of the Laplacian operator satisfy $\sum_{i} \varphi_{i}^{2} \equiv C$, where $C$ is a nonzero constant. Letting

$$
\mu=\inf \{\lambda \in \mathbb{R} \mid \nabla \nabla f \leq \lambda g\}
$$

then we have

(i) $\left(M^{n}, g\right)$ is a shrinking Ricci soliton;

(ii) $B(p, r)$ is isometric to a geodesic ball $B_{K}\left(p^{\prime}, r\right)$ in the space form $\left(M_{K}^{n}, g_{K}\right)$ with constant sectional curvature

$$
K=-[\mu+\varepsilon / 2] / n-1 .
$$

Furthermore, for the complete case, we also have the following similar result:

Theorem 1.13. Let $\left(M^{n}, g\right)$ be a complete gradient Ricci soliton, and suppose that there exists a geodesic ball $B(p, r)$ with center $p$ and radius $r$ such that the eigenfunctions $\left\{\varphi_{i}\right\}_{i=1}^{N}$ corresponding to the first Dirichlet eigenvalue $\lambda_{1}^{D}(B(p, r))$ of the Laplacian operator satisfy $\sum_{i} \varphi_{i}^{2} \equiv C$, where $C$ is a nonzero constant. If

$$
\nabla \nabla f(x) \leq \mu g(x)
$$


for any $x \in B_{K}\left(p^{\prime}, r\right)$ and

$$
\lambda_{1}^{D}\left(B_{K}\left(p^{\prime}, r\right)\right) \leq-\frac{1}{1-1 / n}\left(\mu+\frac{\varepsilon}{2}\right),
$$

where $B_{K}\left(p^{\prime}, r\right)$ is a geodesic ball in the space form $\left(M_{K}^{n}, g_{K}\right)$ with constant sectional curvature $K=-(\mu+\varepsilon / 2) / n-1$, then $B(p, r)$ is isometric to $B_{K}\left(p^{\prime}, r\right)$.

Remark 1.14. Note that any Einstein manifold is automatically a Ricci soliton with a constant Ricci potential function, the similar result is also true naturally.

Corollary 1.15 (Einstein manifold). Let $\left(M^{n}, g\right)$ be a complete Einstein manifold, and suppose that there exists a geodesic ball $B(p, r)$ with center $p$ and radius $r$ such that the eigenfunctions $\left\{\varphi_{i}\right\}_{i=1}^{N}$ corresponding to the first Dirichlet eigenvalue $\lambda_{1}^{D}(B(p, r))$ of the Laplacian operator satisfy $\sum_{i} \varphi_{i}^{2} \equiv C$, where $C$ is a nonzero constant. If

$$
\lambda_{1}^{D}\left(B_{K}\left(p^{\prime}, r\right)\right) \leq-\frac{1}{1-1 / n}\left(\mu+\frac{\varepsilon}{2}\right),
$$

where $B_{K}\left(p^{\prime}, r\right)$ is a geodesic ball in the space form $\left(M_{K}^{n}, g_{K}\right)$ with constant sectional curvature $K=-(\mu+\varepsilon / 2) / n-1$, then $B(p, r)$ is isometric to $B_{K}\left(p^{\prime}, r\right)$.

Remark 1.16. For an Einstein manifold, condition (6) in Corollary 1.13 is not satisfied naturally, but for an Einstein manifold with constant positive scalar curvature, condition (6) is satisfied naturally. In fact, for an Einstein manifold with constant positive scalar curvature, according to the Bonnet-Myers theorem [6], we have the manifold is compact. Moreover, using the lower bound estimation for the first eigenvalue and the equality condition in Theorem 1.1, it can be seen that condition (6) is only satisfied for an Einstein manifold with positive constant scalar curvature.

For the space form $M_{K}$ satisfying $\lambda_{1}\left(M_{K}\right)=n K$, condition (6) is satisfied naturally. Moreover, for an Einstein manifold with positive scalar curvature, condition (6) is also satisfied naturally. Thus, Corollary 1.13 is actually a sufficient condition for the Einstein manifolds isometric isomorphic to the space form. 
On the other hand, the classical Myers theorem says that a complete Riemannian manifold with Ricci curvature bounded below by a positive constant is compact. Based on the study of Ricci flow, Hamilton conjectured (see [6]) that, if $\left(M^{3}, g\right)$ is a complete Riemannian 3manifold with the Ricci pinching condition $R c(g) \geq \varepsilon R g$, where $R>0$ is the positive scalar curvature and $\varepsilon>0$ is a uniform constant, then $M^{3}$ is compact.

With the extra assumption that the sectional curvature is bounded and non-negative (and in this case one has the injectivity radius bounded and Hamilton's Harnack differential inequality for Ricci flow), the conjecture has been proved by Chen and Zhu in [2]. Moreover, the complex version is a conjecture due to Yau (see [10]), and the above question was posed to Hamilton by Shi, who was asked for this by Yau.

In this paper, we will give a partial proof of this conjecture. In fact, we present a characterization of the compactness of manifolds by the first eigenfunctions of the Laplacian operator, and our result also satisfies for general $n$-dim manifolds:

Theorem 1.17 (Main result III). Let $\left(M^{n}, g\right)$ be an $n$-dim complete Riemannian manifold with the Ricci pinching condition $R c(g) \geq \varepsilon R g$, where $R>0$ is the positive scalar curvature and $\varepsilon>0$ is a uniform constant. If there exist some non-constant eigenfunctions $\varphi_{i}$, where $1 \leq i \leq N$, corresponding to the first eigenvalue of the Laplacian operator such that $\sum_{i} \varphi_{i}^{2} \equiv C$, where $C$ is a nonzero constant, then $\left(M^{n}, g\right)$ is compact.

The paper is organized as follows. In Section 2, we prove Theorem 1.4 by using Theorem 1.2. In Section 3, we give the proof of Theorem 1.5 by using Theorem 1.2. Based on these, in Section 4 we prove Theorems 1.11 and 1.12. In Section 5, we present the proof of Theorem 1.14.

2. The comparison theorem for the Schrödinger operator. In this section, we prove the comparison theorem for the Schrödinger operator as follows. 
Proof of Theorem 1.4. Let $\varphi$ be the eigenfunction corresponding to the first Dirichlet eigenvalue $\lambda_{1}^{D}(B(p, r))$ of the Laplacian operator for $B(p, r)$, and let $\varphi_{K}$ be the one corresponding to the first Dirichlet eigenvalue $\mu_{1}^{D}\left(B_{K}\left(p^{\prime}, r\right)\right)$ of the Schrödinger operator for $B_{K}\left(p^{\prime}, r\right)$. Then, by the definition of the first Dirichlet eigenvalue, we have

$$
\lambda_{1}^{D}(B(p, r))=\frac{\int_{B(p, r)}|\nabla \varphi|^{2} d \mu}{\int_{B(p, r)} \varphi^{2} d \mu}
$$

and

$$
\begin{aligned}
\mu_{1}^{D}\left(B_{K}\left(p^{\prime}, r\right)\right) & =\frac{\int_{B_{K}\left(p^{\prime}, r\right)}\left(\left|\nabla \varphi_{K}\right|^{2}+V_{K} \varphi_{K}^{2}\right) d \mu}{\int_{B_{K}\left(p^{\prime}, r\right)} \varphi_{K}^{2} d \mu} \\
& =\frac{\int_{B_{K}\left(p^{\prime}, r\right)}\left|\nabla \varphi_{K}\right|^{2} d \mu}{\int_{B_{K}\left(p^{\prime}, r\right)} \varphi_{K}^{2} d \mu}+\frac{\int_{B_{K}\left(p^{\prime}, r\right)} V_{K} \varphi_{K}^{2} d \mu}{\int_{B_{K}\left(p^{\prime}, r\right)} \varphi_{K}^{2} d \mu}
\end{aligned}
$$

where $\varphi \in \stackrel{o}{H}_{1}^{2}(B(p, r))$ and $\varphi_{K} \in \stackrel{o}{H_{1}^{2}}\left(B_{K}\left(p^{\prime}, r\right)\right)$.

Then, by the definition of the first Dirichlet eigenvalue, we have

$$
\begin{aligned}
\mu_{1}^{D}(B(p, r)) & =\inf _{\substack{o \\
\phi H_{1}^{2}(B(p, r))}} \frac{\int_{B(p, r)}\left(|\nabla \phi|^{2}+V \phi^{2}\right) d \mu}{\int_{B(p, r)} \phi^{2} d \mu} \\
& \leq \frac{\int_{B(p, r)}|\nabla \varphi|^{2} d \mu}{\int_{B(p, r)} \varphi^{2} d \mu}+\frac{\int_{B(p, r)} V \varphi^{2} d \mu}{\int_{B(p, r)} \varphi^{2} d \mu} \\
& \leq \lambda_{1}^{D}(B(p, r))+\max _{x \in B(p, r)} V(x)
\end{aligned}
$$

and

$$
\begin{aligned}
\mu_{1}^{D}\left(B_{K}\left(p^{\prime}, r\right)\right) & =\frac{\int_{B_{K}\left(p^{\prime}, r\right)}\left|\nabla \varphi_{K}\right|^{2} d \mu}{\int_{B_{K}\left(p^{\prime}, r\right)} \varphi_{K}^{2} d \mu}+\frac{\int_{B_{K}\left(p^{\prime}, r\right)} V_{K} \varphi_{K}^{2} d \mu}{\int_{B_{K}\left(p^{\prime}, r\right)} \varphi_{K}^{2} d \mu} \\
& \geq \inf _{\phi \in H_{1}^{2}\left(B_{K}\left(p^{\prime}, r\right)\right)} \frac{\int_{B_{K}\left(p^{\prime}, r\right)}|\nabla \phi|^{2} d \mu}{\int_{B_{K}\left(p^{\prime}, r\right)} \phi^{2} d \mu}+\min _{x \in B_{K}\left(p^{\prime}, r\right)} V_{K}(x) \\
& =\lambda_{1}^{D}\left(B_{K}\left(p^{\prime}, r\right)\right)+\min _{x \in B_{K}\left(p^{\prime}, r\right)} V_{K}(x) .
\end{aligned}
$$


Thus,

$$
\begin{gathered}
\mu_{1}^{D}(B(p, r))-\max _{x \in B(p, r)} V(x) \leq \lambda_{1}^{D}(B(p, r)), \\
\lambda_{1}^{D}\left(B_{K}\left(p^{\prime}, r\right)\right) \leq \mu_{1}^{D}\left(B_{K}\left(p^{\prime}, r\right)\right)-\min _{x \in B_{K}\left(p^{\prime}, r\right)} V_{K}(x) .
\end{gathered}
$$

Note that $R c \geq(n-1) K g$; thus, by Theorem 1.2 , we have

$$
\lambda_{1}^{D}(B(p, r)) \leq \lambda_{1}^{D}\left(B_{K}\left(p^{\prime}, r\right)\right) .
$$

Together with equation (7), it follows that

$$
\mu_{1}^{D}(B(p, r))-\max _{x \in B(p, r)} V(x) \leq \mu_{1}^{D}\left(B_{K}\left(p^{\prime}, r\right)\right)-\min _{x \in B_{K}\left(p^{\prime}, r\right)} V_{K}(x),
$$

which implies that

$$
\mu_{1}^{D}(B(p, r)) \leq \mu_{1}^{D}\left(B_{K}\left(p^{\prime}, r\right)\right)+\max _{x \in B(p, r)} V(x)-\min _{x \in B_{K}\left(p^{\prime}, r\right)} V_{K}(x) .
$$

Furthermore, if the equality in equation (8) holds, by the proof we have

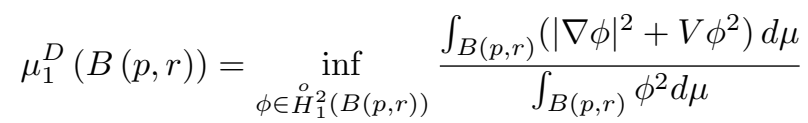

$$
\begin{aligned}
& =\frac{\int_{B(p, r)}\left(|\nabla \varphi|^{2}+V \varphi^{2}\right) d \mu}{\int_{B(p, r)} \varphi^{2} d \mu} \\
& \frac{\int_{B_{K}\left(p^{\prime}, r\right)}\left|\nabla \varphi_{K}\right|^{2} d \mu}{\int_{B_{K}\left(p^{\prime}, r\right)} \varphi_{K}^{2} d \mu}=\inf _{\phi \in H_{1}^{2}\left(B_{K}\left(p^{\prime}, r\right)\right)} \frac{\int_{B_{K}\left(p^{\prime}, r\right)}|\nabla \phi|^{2} d \mu}{\int_{B_{K}\left(p^{\prime}, r\right)} \phi^{2} d \mu} \\
& =\lambda_{1}^{D}\left(B_{K}\left(p^{\prime}, r\right)\right), \\
& V(x)=\max _{x \in B(p, r)} V(x), \\
& V_{K}(x)=\min _{x \in B_{K}\left(p^{\prime}, r\right)} V_{K}(x)
\end{aligned}
$$

and

$$
\lambda_{1}^{D}(B(p, r))=\lambda_{1}^{D}\left(B_{K}\left(p^{\prime}, r\right)\right)
$$

Hence, $\varphi$ is also an eigenfunction corresponding to the first Dirichlet eigenvalue $\mu_{1}^{D}(B(p, r))$ of the Schrödinger operator, $\varphi_{K}$ is also an eigenfunction corresponding to the first Dirichlet eigenvalue $\lambda_{1}^{D}\left(B_{K}\left(p^{\prime}, r\right)\right)$ 
of the Laplacian operator. The functions $V(x)$ and $V_{K}(x)$ are constant on $B(p, r)$ and $B_{K}\left(p^{\prime}, r\right)$, respectively.

Moreover by using the equality condition in Theorem 1.2, it follows from

$$
\lambda_{1}^{D}(B(p, r))=\lambda_{1}^{D}\left(B_{K}\left(p^{\prime}, r\right)\right)
$$

that $B(p, r)$ is isometric to $B_{K}\left(p^{\prime}, r\right)$.

Conversely, if $B(p, r)$ is isometric to $B_{K}\left(p^{\prime}, r\right)$, the eigenfunctions corresponding to the first Dirichlet eigenvalue $\lambda_{1}^{D}(B(p, r))$ and $\lambda_{1}^{D}\left(B_{K}\left(p^{\prime}, r\right)\right)$ of the Laplacian operator are, respectively, the eigenfunctions corresponding to the first Dirichlet eigenvalue $\mu_{1}^{D}(B(p, r))$ and $\mu_{1}^{D}\left(B_{K}\left(p^{\prime}, r\right)\right)$ of the Schrödinger operator. Together with the condition that $V(x)$ and $V_{K}(x)$ are constant on $B(p, r)$ and $B_{K}\left(p^{\prime}, r\right)$, respectively, the equality in equation (2.2) holds clearly.

3. Proof of Theorem 1.5. In this section, we prove Theorem 1.5.

Proof of Theorem 1.5. Firstly, select two points $p$ and $q$ such that

$$
d(p, q)=d_{M_{K}^{n}}=\frac{\pi}{\sqrt{K}}
$$

and consider the geodesic balls $B_{K}\left(p,(\pi /(2 \sqrt{K}))\right.$ and $B_{K}(q,(\pi /(2 \sqrt{K})))$ in the $n$-dim simply connected space form $M_{K}^{n}$. We denote $u$ and $v$ as the first Dirichlet eigenfunctions of the Laplacian operator corresponding to $B_{K}(p,(\pi /(2 \sqrt{K})))$ and $B_{K}(q,(\pi /(2 \sqrt{K})))$, respectively, and define the following two functions:

$$
\widetilde{u}(x)= \begin{cases}u(x) & x \in B_{K}(p,(\pi /(2 \sqrt{K}))), \\ 0 & x \in M_{K}^{n} \backslash B_{K}(p,(\pi /(2 \sqrt{K}))),\end{cases}
$$

and

$$
\widetilde{v}(x)= \begin{cases}v(x) & x \in B_{K}(q,(\pi /(2 \sqrt{K}))), \\ 0 & x \in M_{K}^{n} \backslash B_{K}(q,(\pi /(2 \sqrt{K}))) .\end{cases}
$$


Then

$$
\frac{\int_{M_{K}^{n}}|\nabla \widetilde{u}|^{2} d \mu}{\int_{M_{K}^{n}} \widetilde{u}^{2} d \mu}=\frac{\int_{B_{K}(p,(\pi /(2 \sqrt{K})))}|\nabla u|^{2} d \mu}{\int_{B_{K}(p,(\pi /(2 \sqrt{K})))} u^{2} d \mu}=\lambda_{1}^{D}\left(B_{K}\left(p, \frac{\pi}{2 \sqrt{K}}\right)\right)
$$

and

$$
\frac{\int_{M_{K}^{n}}|\nabla \widetilde{v}|^{2} d \mu}{\int_{M_{K}^{n}} \widetilde{v}^{2} d \mu}=\frac{\int_{B_{K}(q,(\pi /(2 \sqrt{K})))}|\nabla v|^{2} d \mu}{\int_{B_{K}(q,(\pi /(2 \sqrt{K})))} v^{2} d \mu}=\lambda_{1}^{D}\left(B_{K}\left(q, \frac{\pi}{2 \sqrt{K}}\right)\right) .
$$

Now we choose a constant $C$ such that

$$
\int_{M_{K}^{n}}(\widetilde{u}+C \widetilde{v}) d \mu=0
$$

and by the definition of the first eigenfunction of the Laplacian operator, we have

$$
\lambda_{1}\left(M_{K}^{n}\right) \leq \frac{\int_{M_{K}^{n}}|\nabla(\widetilde{u}+C \widetilde{v})|^{2} d \mu}{\int_{M_{K}^{n}}(\widetilde{u}+C \widetilde{v})^{2} d \mu}
$$

Note that $M_{K}^{n}$ is a space form with diameter $d_{M_{K}^{n}}=\pi / \sqrt{K}$, and it follows that

$$
\lambda_{1}^{D}\left(B_{K}\left(p, \frac{\pi}{2 \sqrt{K}}\right)\right)=\lambda_{1}^{D}\left(B_{K}\left(q, \frac{\pi}{2 \sqrt{K}}\right)\right)
$$

and

$$
\operatorname{Vol}\left(B_{K}\left(p, \frac{\pi}{2 \sqrt{K}}\right) \cap B_{K}\left(q, \frac{\pi}{2 \sqrt{K}}\right)\right)=0
$$

Then, by using equations (9) and (10), we have

$$
\begin{aligned}
\lambda_{1}\left(M_{K}^{n}\right) & \leq \frac{\int_{M_{K}^{n}}|\nabla(\widetilde{u}+C \widetilde{v})|^{2} d \mu}{\int_{M_{K}^{n}}(\widetilde{u}+C \widetilde{v})^{2} d \mu}=\frac{\int_{M_{K}^{n}}|\nabla \widetilde{u}|^{2} d \mu+C^{2} \int_{M_{K}^{n}}|\nabla \widetilde{v}|^{2} d \mu}{\int_{M_{K}^{n}} \widetilde{u}^{2} d \mu+C^{2} \int_{M_{K}^{n}} \widetilde{v}^{2} d \mu} \\
& =\lambda_{1}^{D}\left(B_{K}\left(p, \frac{\pi}{2 \sqrt{K}}\right)\right)=\lambda_{1}^{D}\left(B_{K}\left(q, \frac{\pi}{2 \sqrt{K}}\right)\right) .
\end{aligned}
$$


For the other inequality, note that the metric of space form $M_{K}^{n}$ is the form of

$$
g_{K}=d r^{2}+s_{K}(r) g_{S^{n-1}} .
$$

Then, by Theorem 1.1, we can select the first eigenfunction of the Laplacian operator

$$
\varphi(x)=A \cos (\sqrt{K} r)+B \sin (\sqrt{K} r)
$$

where $r=d(p, x)$ such that

$$
\begin{aligned}
\lambda_{1}\left(M_{K}^{n}\right) & =\frac{\int_{M_{K}^{n}}|\nabla \varphi|^{2} d \mu}{\int_{M_{K}^{n}} \varphi^{2} d \mu}=\frac{\int_{0}^{\pi / \sqrt{K}}|\nabla \varphi(r)|^{2} s_{K}(r)^{n-1} d r \int_{S^{n-1}} d \mu_{S^{n-1}}}{\int_{0}^{\pi / \sqrt{K}} \varphi(r)^{2} s_{K}(r)^{n-1} d r \int_{S^{n-1}} d \mu_{S^{n-1}}} \\
& =\frac{\int_{0}^{\pi / \sqrt{K}}|\nabla \varphi(r)|^{2} s_{K}(r)^{n-1} d r}{\int_{0}^{\pi / \sqrt{K}} \varphi(r)^{2} s_{K}(r)^{n-1} d r} \\
& =\lim _{s \rightarrow \pi / \sqrt{K}} \frac{\int_{0}^{s}|\nabla \varphi(r)|^{2} s_{K}(r)^{n-1} d r}{\int_{0}^{s} \varphi(r)^{2} s_{K}(r)^{n-1} d r}
\end{aligned}
$$

where we use the fact that $M_{K}^{n}$ is a space form with diameter $d_{M_{K}^{n}}=$ $\pi / \sqrt{K}$. Then we define another function $\varphi_{s} \in H_{1}^{2}\left(B_{K}(p, s)\right)$ such that $\varphi_{s}(x)=\varphi(x)$ for any $x \in B_{K}(p, s)$, and, by the definition of the first Dirichlet eigenvalue of the Laplacian operator for $B_{K}(p, s)$, it follows that

$$
\begin{aligned}
\frac{\int_{0}^{s}|\nabla \varphi(r)|^{2} s_{K}(r)^{n-1} d r}{\int_{0}^{s} \varphi(r)^{2} s_{K}(r)^{n-1} d r} & =\frac{\int_{0}^{s}\left|\nabla \varphi_{s}(r)\right|^{2} s_{K}(r)^{n-1} d r \int_{S^{n-1}} d \mu_{S^{n-1}}}{\int_{0}^{s} \varphi_{s}(r)^{2} s_{K}(r)^{n-1} d r \int_{S^{n-1}} d \mu_{S^{n-1}}} \\
& =\frac{\int_{B_{K}(p, s)\left|\nabla \varphi_{s}\right|^{2} d \mu} \geq \lambda_{1}^{D}\left(B_{K}(p, s)\right) .}{\int_{B_{K}(p, s)} \varphi_{s}^{2} d \mu} .
\end{aligned}
$$

Consequently, by the definition of $\lambda_{1}^{D}\left(M_{K}^{n}\right)$, we have

$$
\begin{aligned}
\lambda_{1}\left(M_{K}^{n}\right) & =\lim _{s \rightarrow \pi / \sqrt{K}} \frac{\int_{0}^{s}|\nabla \varphi(r)|^{2} s_{K}(r)^{n-1} d r}{\int_{0}^{s} \varphi(r)^{2} s_{K}(r)^{n-1} d r} \geq \lim _{s \rightarrow \pi / \sqrt{K}} \lambda_{1}^{D}\left(B_{K}(p, s)\right) \\
& =\lambda_{1}^{D}\left(M_{K}^{n}\right) .
\end{aligned}
$$


4. Characterizations of Ricci solitons as space forms. In this section, by using Theorems 1.1, 1.2 and 1.5, we turn to prove Theorems 1.11 and 1.12 .

Proof of Theorem 1.11 .

(i) If $\left(M^{n}, g\right)$ is a steady or expanding Ricci soliton

$$
R c+\nabla \nabla f+\frac{\varepsilon}{2} g=0
$$

where $\varepsilon \geq 0$, then, by Theorem 1.9 , we have $M^{n}$ is actually an Einstein manifold with the Ricci potential function $f$ being a constant. Thus,

$$
R c=-\frac{\varepsilon}{2} g \leq 0,
$$

which leads to a contradiction to the positive Ricci curvature.

(ii) By the assumption of Theorem 1.11, for the geodesic ball $B(p, r)$, we have

$$
\Delta \varphi_{i}+\lambda_{1}^{D}(B(p, r)) \varphi_{i}=0
$$

and

$$
\sum_{i} \varphi_{i}^{2} \equiv C
$$

where $1 \leq i \leq N$ for some $N \in \mathbb{N}$. Thus,

$$
\begin{aligned}
0 & =\Delta\left(\sum_{i} \varphi_{i}^{2}\right)=\sum_{i} 2\left|\nabla \varphi_{i}\right|^{2}+2 \sum_{i} \varphi_{i} \Delta \varphi_{i} \\
& =\sum_{i} 2\left|\nabla \varphi_{i}\right|^{2}-2 \lambda_{1}^{D}(B(p, r)) \sum_{i} \varphi_{i}^{2},
\end{aligned}
$$

which implies that

$$
\sum_{i}\left|\nabla \varphi_{i}\right|^{2}=C \lambda_{1}^{D}(B(p, r))
$$

Taking the Laplacian of both sides of equation (4.1) and using the Bochner formula [6]:

$$
\Delta|\nabla f|^{2}=2|\nabla \nabla f|^{2}+2\langle\nabla f, \nabla \Delta f\rangle+2 R c(\nabla f, \nabla f),
$$

we have 


$$
\begin{aligned}
0= & \sum_{i} \Delta\left|\nabla \varphi_{i}\right|^{2} \\
= & 2 \sum_{i}\left|\nabla \nabla \varphi_{i}\right|^{2}+2 \sum_{i}\left\langle\nabla \varphi_{i}, \nabla \Delta \varphi_{i}\right\rangle+2 \sum_{i} R c\left(\nabla \varphi_{i}, \nabla \varphi_{i}\right) \\
= & 2 \sum_{i}\left|\nabla \nabla \varphi_{i}\right|^{2}-2 \lambda_{1}^{D}(B(p, r))^{2} \sum_{i} \varphi_{i}^{2} \\
& -2 \sum_{i} \nabla \nabla f\left(\nabla \varphi_{i}, \nabla \varphi_{i}\right)-\varepsilon \sum_{i}\left|\nabla \varphi_{i}\right|^{2} \\
\geq & \frac{2}{n} \sum_{i}\left|\Delta \varphi_{i}\right|^{2}-2 \lambda_{1}^{D}(B(p, r))^{2} \sum_{i} \varphi_{i}^{2} \\
& -2 \mu \sum_{i}\left|\nabla \varphi_{i}\right|^{2}-\varepsilon \sum_{i}\left|\nabla \varphi_{i}\right|^{2} \\
= & -2\left(1-\frac{1}{n}\right) \lambda_{1}^{D}(B(p, r))^{2} C-2\left(\mu+\frac{\varepsilon}{2}\right) \lambda_{1}^{D}(B(p, r)) C .
\end{aligned}
$$

Note that, if for any geodesic ball $B(p, r)$, the first Dirichlet eigenvalue $\lambda_{1}^{D}(B(p, r))$ of the Laplacian operator is positive, then together with $C>0$ we have

$$
\lambda_{1}^{D}(B(p, r)) \geq-\frac{1}{(1-1 / n)}\left(\mu+\frac{\varepsilon}{2}\right) .
$$

On the other hand, since $\left(M^{n}, g\right)$ is a compact gradient Ricci soliton with positive Ricci curvature, we have

$$
0<\delta g \leq R c=-\left(\nabla \nabla f+\frac{\varepsilon}{2} g\right)
$$

which is equivalent to

$$
\nabla \nabla f \leq-\left(\delta+\frac{\varepsilon}{2}\right) g .
$$

By the definition of $\mu$, we have $\mu \leq-(\delta+\varepsilon / 2)$. Then

$$
R c=-\nabla \nabla f-\frac{\varepsilon}{2} g \geq-\left(\mu+\frac{\varepsilon}{2}\right) g \geq \delta g>0 .
$$


Thus, for the space form $\left(M_{K}^{n}, g_{K}\right)$ with constant sectional curvature $K=-[\mu+\varepsilon / 2] / n-1$, we have

$$
R c\left(g_{K}\right)=-\left(\mu+\frac{\varepsilon}{2}\right) g>0 .
$$

Then, by the equality condition in Theorem 1.1, we have

$$
\lambda_{1}\left(M_{K}^{n}\right)=-\frac{1}{(1-1 / n)}\left(\mu+\frac{\varepsilon}{2}\right) .
$$

Furthermore, by Theorem 1.2, it follows from equation (4.3) that

$$
\lambda_{1}^{D}(B(p, r)) \leq \lambda_{1}^{D}\left(B_{K}\left(p^{\prime}, r\right)\right),
$$

where $B_{K}\left(p^{\prime}, r\right)$ is a geodesic ball in the space form $M_{K}^{n}$ with $K=$ $-[\mu+\varepsilon / 2] / n-1$. Note that Theorem 1.5 implies that

$$
\lambda_{1}^{D}\left(B_{K}\left(p^{\prime}, r\right)\right) \leq \lambda_{1}\left(M_{K}^{n}\right)=-\frac{1}{(1-1 / n)}\left(\mu+\frac{\varepsilon}{2}\right),
$$

where $\lambda_{1}\left(M_{K}^{n}\right)$ denote the first eigenvalue of the Laplacian operator for the space form $M_{K}^{n}$.

Thus, by equations (4.2) and (4.5), it follows that

$$
\lambda_{1}^{D}(B(p, r)) \geq-\frac{1}{(1-1 / n)}\left(\mu+\frac{\varepsilon}{2}\right) \geq \lambda_{1}^{D}\left(B_{K}\left(p^{\prime}, r\right)\right),
$$

Together with equation (4.4), we have

$$
\lambda_{1}^{D}(B(p, r))=\lambda_{1}^{D}\left(B_{K}\left(p^{\prime}, r\right)\right) .
$$

Then, by the equality condition in Theorem 1.2 , we derive that $B(p, r)$ is isometric to $B_{K}\left(p^{\prime}, r\right)$.

Proof of Theorem 1.12. As in the proof of Theorem 1.11, for the geodesic ball $B(p, r)$, we can derive that

$$
\lambda_{1}^{D}(B(p, r)) \geq-\frac{1}{(1-1 / n)}\left(\mu+\frac{\varepsilon}{2}\right) .
$$

Then, together with the assumption

$$
\lambda_{1}^{D}\left(B_{K}\left(p^{\prime}, r\right)\right) \leq-\frac{1}{(1-1 / n)}\left(\mu+\frac{\varepsilon}{2}\right),
$$


we can also obtain that

$$
\lambda_{1}^{D}(B(p, r)) \geq \lambda_{1}^{D}\left(B_{K}\left(p^{\prime}, r\right)\right),
$$

and the rest of the proof is similar to the proof of Theorem 1.11.

\section{Compactness of complete manifolds with Ricci pinching.} In this section, we prove Theorem 1.14.

Proof of Theorem 1.14. As the proof of Theorem 1.11, we can also derive

$$
\begin{aligned}
0 & =2 \sum_{i}\left|\nabla \nabla \varphi_{i}\right|^{2}-2 \lambda_{1}^{2}\left(M^{n}\right) \sum_{i} \varphi_{i}^{2}+2 \sum_{i} R c\left(\nabla \phi_{i}, \nabla \phi_{i}\right) \\
& \geq \frac{2}{n} \sum_{i}\left|\Delta \varphi_{i}\right|^{2}-2 \lambda_{1}^{2}\left(M^{n}\right) \sum_{i} \varphi_{i}^{2}+2 \varepsilon R \sum_{i}\left|\nabla \varphi_{i}\right|^{2} \\
& =-2\left(1-\frac{1}{n}\right) \lambda_{1}^{2}\left(M^{n}\right) C+2 C \varepsilon R \lambda_{1}\left(M^{n}\right) .
\end{aligned}
$$

If $\lambda_{1}\left(M^{n}\right)$ is equal to 0 , then by $\mathrm{Li}$ and Yau's gradient estimate (see [9]), we derive that $\varphi_{i} \equiv C_{i}$, where $C_{i}$ is a constant for each $1 \leq i \leq N$. Thus, without loss of generality, we can assume that $\lambda_{1}\left(M^{n}\right)>0$, and consequently,

$$
\lambda_{1}\left(M^{n}\right) \geq \frac{n \varepsilon}{n-1} R
$$

Then we consider the geodesic ball $B(p, r)$ with center $p$ and radius $r$, and, by Corollary 1.3, we have

$$
\lambda_{1}^{D}(B(p, r)) \leq \frac{C_{n}}{d_{B(p, r)}^{2}},
$$

where $C_{n}=2 n(n+4)$ and $d_{B(p, r)}$ is the diameter of $B(p, r)$.

On the other hand, let $\varphi$ be the first Dirichlet eigenfunction of the Laplacian operator for $B\left(p, r_{1}\right)$ such that

$$
\int_{B\left(p, r_{1}\right)} \varphi^{2} d \mu=1 .
$$


Then, for a larger geodesic ball $B\left(p, r_{2}\right)$, it is easy to see that

$$
\widetilde{\varphi}(x)= \begin{cases}\varphi(x) & x \in B\left(p, r_{1}\right) \\ 0 & x \in B\left(p, r_{2}\right) \backslash B\left(p, r_{1}\right)\end{cases}
$$

is a function defined on $B\left(p, r_{2}\right)$ such that

$$
\int_{B\left(p, r_{2}\right)} \widetilde{\varphi}^{2} d \mu=1 .
$$

Then, by the definition of the first Dirichlet eigenvalue of the Laplacian operator, we have

$$
\begin{aligned}
\lambda_{1}\left(B\left(p, r_{1}\right)\right) & =\int_{B\left(p, r_{1}\right)}|\nabla \varphi|^{2} d \mu=\int_{B\left(p, r_{2}\right)}|\nabla \widetilde{\varphi}|^{2} d \mu \\
& \geq \inf _{\|\phi\|_{2}=1} \int_{B\left(p, r_{2}\right)}|\nabla \phi|^{2} d \mu=\lambda_{1}\left(B\left(p, r_{2}\right)\right) .
\end{aligned}
$$

Thus, if the first eigenvalue of the Laplacian operator for $M^{n}$

$$
\lambda_{1}\left(M^{n}\right)=\lim _{r \rightarrow \infty} \lambda_{1}^{D}(B(p, r))
$$

exists, then actually $\lambda_{1}^{D}(B(p, r))$ tends to $\lambda_{1}\left(M^{n}\right)$ monotone decreasingly. Hence,

$$
\frac{C_{n}}{d_{B(p, r)}^{2}} \geq \lambda_{1}^{D}(B(p, r)) \geq \lambda_{1}\left(M^{n}\right) \geq \frac{n \varepsilon}{n-1} R(p),
$$

for any $r$, that is to say, that

$$
d_{B(p, r)} \leq \sqrt{\frac{2(n+4)(n-1)}{\varepsilon R(p)}}<\infty,
$$

for any $r$, where we use $R(p)>0$. This implies that the manifold $M^{n}$ is compact.

Acknowledgments. We would especially like to thank the referee for meaningful suggestions that led to improvements of the article.

\section{REFERENCES}

1. C. Böhm and B. Wilking, Manifolds with positive curvature operators are space forms, Ann. Math. 167 (2008), 1079-1097. 
2. B.L. Chen and X.P. Zhu, Complete Riemannian manifolds with pointwise pinched curvature, Invent. Math. 140 (2000), 423-452.

3. S.Y. Cheng, Eigenvalue comparison theorems and its geometric application, Math. Z. 143 (1975), 289-297.

4. A characterization of the 2-sphere by eigenfunctions, Proc. Amer. Math. Soc. 55 (1976), 379-381.

5. N Nodal sets and eigenfunctions, Comm. Math. Helv. 51 (1976), 43-55.

6. B. Chow, P. Lu and L. Ni, Hamilton's Ricci flow, Lect. Contemp. Math. 3, Sci. Press Grad. Stud. Math. 77, American Mathematical Society (co-publication), 2006.

7. R.S. Hamilton, Four manifolds with positive curvature operator, J. Diff. Geom. 24 (1986), 153-179.

8. The Harnack estimate for the Ricci flow, J. Diff. Geom. 37 (1993), 225-243.

9. R. Schoen and S.T. Yau, Lectures on differential geometry, Conference Proceedings and Lecture Notes in Geometry and Topology I, International Press, Cambridge, MA, 1994.

10. W.X. Shi and S.T. Yau, A note on the total curvature of a Kahler manifold, Math. Res. Lett. 3 (1996), 123-132.

11. S. Tachibana, A theorem of Riemannian manifolds of positive curvature operator, Proc. Japan Acad. 50 (1974), 301-302.

School of Mathematical Sciences, Ocean University of China, Lane 238, Songling Road, Laoshan District, Qingdao City, Shandong Province, 266100, P.R. CHINA

Email address: gaoxiangshuli@126.com 